\title{
Organ on Chip Development for The Improvement of New Smart Cancer Therapeutic Carriers Study
}

\author{
Eric Gaffet ${ }^{1}$ and Halima Alem ${ }^{1,2 *}$ \\ ${ }^{1}$ Jean Lamour Institute (UMR 7198), France \\ ${ }^{2}$ University Institute of France (IUF), France
}

*Corresponding author: Halima Alem, Université de Lorraine, CNRS, Campus Artem 2 allée André Guinier - BP 50840, F54011 Nancy Cedex, France

\section{ARTICLE INFO}

Received: 幽 November 13, 2019

Published: 慧 November 19, 2019

Citation: Eric Gaffet, Halima Alem. Organ on Chip Development for The Improvement of New Smart Cancer Therapeutic Carriers Study. Biomed J Sci \& Tech Res 23(1)-2019. BJSTR. MS.ID.003841.

\begin{abstract}
There is a real need nowadays for the improvement of our knowledge in predictive medicine in the frame of the development responsive nanocarrier for cancer therapy. One way to achieve it, is to develop an innovative approach based on organ on chip (OOC) devices for the evaluation and the enhancement of the therapeutic efficiency of smart nano-object. Those devices will not only significantly help in providing a new insight of the main cause of the low efficiency of cancer therapeutic nano-carrier when translated to human but also to develop promising new nano-carrier with the optimal functionality and therapeutic efficiency.
\end{abstract}

Keywords: Superparamagnetic Iron Oxide Nanoparticles; Food and Drug Administration; Magnetic Resonance Imaging; Organ on chip

\section{Mini Review}

\section{Development of targeting drug nano carrier for cancer therapy/theragnostic}

One of the major challenges in nanomedicine is to develop nanoparticulate systems able to serve as efficient diagnostic, therapeutic or theragnostic tools against severe diseases, such as infectious or neurodegenerative disorders [1]. In the frame of cancer therapy, efforts are currently devoted in the development of theragnostic agents that can diagnose, image and treat at the same time a tumor. Those agents are mainly composed of nano-sized systems (i.e. nanoparticles, nano-rods, nanotubes, ...) that have shown promising results which lead to their selection for clinical investigation [2,3]. Most common nano systems are nanoparticulate systems owing to their ease of synthesis, high specific surface area to volume ratio, which open various possibility in their functionalization. Those nano-objects can be directly functionalized by a targeting ligand to enhance the uptake, and combined with a polymer for stealth and drug loading-release properties $[2,4]$

Superparamagnetic nanoparticles (NPs), among them, superparamagnetic iron oxide nanoparticles (SPIONs) that have received a great attention since their development 20 years ago5, as a liver contrasting agent. Their properties, originating from the nanosized dimension and shape, have been found to allow different biodistribution and opportunities compared to the conventional molecular imaging agents. Moreover, they do not retain their magnetization once the magnetic field is removed [5]. It is worth to mention that SPIONs are already approved by the US Food and Drug Administration (FDA) and by the European Union [4] and have been greatly investigated due to their good biocompatibility, low toxicity and ease of synthesis [6]. Moreover, for theragnostic application, those nanoparticles have gained great attention owing to their hyperthermia properties [6]. Hyperthermia results from the ability of the SPIONS to generate heat upon application of a local high frequency alternating magnetic field [7]. For biological application of those very fascinating nanoparticles, efforts have then been concentrated in their surface functionalization not only to enhance their water solubility and stability but also to give them stealth and cancer cells targeting properties [1-8].

Different studies have enlightened first that the NPs properties as size, geometry, surface chemistry, elasticity, stiffness, targeting 
ligands, can influence the biological response, and thus the therapeutic outcomes [3-10]. Secondly there is no doubt that by combining diagnostic and therapeutic function in the same object, this so-called theragnostic nano medicine is paving the way for the monitoring and the enhancement of the treatment. It must be nevertheless pointed out that most of the current studies of the NPs in-vivo behavior are based on animals' data, and the understanding of their behavior in human body is still slow and lack due to obvious ethic limitations [2]. Indeed, despite the development of numerous studies related to the pharmacokinetic properties of the nanoobjects across species in pre-clinical and clinical studies, very few have allowed the establishment of correlation between the NPs toxicity and the efficiency across the species to be translated to human [2].

Such a feature can be explained by the fact that when considering the injection of NPs in the body, they must face to their rapid covering by various proteins (so-called protein corona, PC) [11]. This PC by changing the particle surface energy, size, cellular uptake, biodistribution, toxicity, can either completely contra modify (enhancement or decrease) the NPs efficiency observed in 2D or 3D in-vitro culture and can evolve from a specie to another but also from a person to another and can vary with the cancer type environment [2-11].

We envision then to study the influence of the NPs chemical functionalization, and to show if our nano systems "undergoes" the PC, or on the contrary, if the PC can be mastered with relevant polymer/biomolecule conjugation that would make us controlling the PC which is nowadays a real challenge to enhance the therapeutic efficiency of the functional nanoparticles. Some works lead the elaboration and the study of non-cytotoxic responsive core/ shell magnetic nanoparticles that can be used as contrast agent in magnetic resonance imaging (MRI). They were obtained from the growth, by controlled living polymerization, of co-polymers based on 2-(2-methoxy) ethyl methacrylate (ME02MA) and oligo (ethylene glycol) methacrylate (OEGMA) moieties which led to $\mathrm{P}($ MEO2MAxOEGMA100-X) (the ratio of the both monomers were varied to tune the DOX release temperature). We have shown that they are able to load and release a model drug, i. e. the doxorubicin (DOX) [9-13] with an associated DOX loading capacity of $15 \%$ which is largely higher than the current nanocarrier performances [3-14]. Additionally, to their release properties, we have demonstrated that their thermo-responsive behavior is completely reversible [915], and their hyperthermia properties were recently studied [16]. In the frame of the development of targeted carrier, it is crucial to enhance the selective binding and the delivery of the drug to the targeted cells via the receptor-mediated endocytosis [16], folic acid (FA) successfully anchored at the periphery of these NPs [12]. A step forward, under work, consists now to transpose the process we have developed, to the introduction of specific antibodies, peptides or ligands for the enhanced endocytosis of the NPs within the cancer cells.

\section{Organ on chip (O0C)}

Considering the elements reported above, we must mention that the studies in 2D cells cultures, but also in-vivo data have shown their limit. The last estimation showed that less than $8 \%$ of successful animal trials for cancer drugs have been translated/ converted into to successful human clinical trials [17]. It appeared then that the need of reliable laboratory models is necessary to mimic the whole cancer cells environment and behavior. Biomimetic microsystems have then shown very fascinating results as they consist of the engineering of microchips that contain human cells that can reconstitute the organ level functions for drug screening, diagnosis and therapeutic application [18-21]. This constitutes the so-called organ on chip (OOC). These sophisticated OOC models are not only the best-developed devices nowadays to overcome the experimentation with animals, but also, they are a real issue for the development of non-expansive diagnostic and therapeutic approaches. It is expected that it would solve many of the important challenges in nanomedicine in the future [21-22].

This last feature allows thinking that by concentrating the effort of interdisciplinary effort at the intersection of engineering, biology, medicine and material science, personalized OOC can be obtained and a direct test of drug on each patient cells could be considered. This is a direct way for personalized medicine as every test could be performed on specific patient cells to lead to a "model" organ, i.e. POOC for Personalized OOC. OOC devices are microfluidic cell cultures, that are fabricated from microsensor technology. They are composed of two continuously perfused compartments :

1. In one compartment, living cells can develop and arrange to create a tissue and organ level physiology,

2. In the second compartment, blood circulation is achieved for the cell's nutrition and communication.

The both compartments are separated by a porous membrane for achieving the cells immobilization and growth and their nutrition [18-23].

A huge advancement in $00 \mathrm{C}$ technology was witnessed this last decade which gave rise to a library of different organs. Nevertheless, the development of polymeric membranes that are essential as the cancer cells are in direct contact with them still lack [24] (the few works that are concentrated on the membranes for OOC are mainly focused on healthy cells). Indeed, the membrane should simulate the cancer cells environment and mimic the architecture of the tissue [25-27]. The preferred membrane nowadays used is based on poly(dimethyl siloxane) (PDMS) as it is flexible (can then withstand deformation for many cycles), transparent in UVVisible spectrum range and can be functionalized by different ways to render it hydrophilic and biocompatible [27-30]. Its induced porosity by different techniques makes it also an ideal candidate for the communication between the both compartments. It is the crucial to develop porous, nanostructured and functionalized PDMS 
based membranes that can display good mechanical properties and functionalities for cancer cells growth for OOC development.

\section{Conclusion}

To summarize, despite the tremendous effort of different research communities, nanomedicine impact on patient still very limited. The major reasons are first the efficiency of the targeting and of the drug loading capacity of the nanomedicine. The second main limitation when considering the nanomedicine release in the body is that its efficacy can be altered by the protein corona that we plan to study and control via the NPs design. Finally, it is well established, that from a body to another, the nanomedicine efficacy can change drastically, that is the reason why it is crucial to take advantage and improvement of the promising POOC devices for personalized medicine for cancer cells by focusing first on the membrane for an optimal cancer cells growth and secondly its implementation to obtain new highly efficient 00C. The establishment of a relationship between the NPs engineering/functionalities and their therapeutic efficiency would pave the way for the development of nano-platform that can be functionalized at will.

\section{References}

1. S Laurent, D Forge, M Port, A Roch, C Robic, et al. (2008) Magnetic iron oxide nanoparticles: synthesis, stabilization, vectorization, physicochemical characterizations, and biological applications Chem Rev 108(6): 2064-2110.

2. J Shi, PW Kantoff, R Wooster, O C Farokhzad (2017) Cancer nanomedicine: progress, challenges and opportunities Nat Rev Cancer 17(1): 20-37.

3. S Mura, J Nicolas, P Couvreur (2013) Stimuli-responsive nanocarriers for drug delivery Nat Mater 12(11): 991-1003.

4. AS Thakor, J V Jokerst, P Ghanouni, J L Campbell, E Mittra, et al. (2016) Med 57: 1833-1837

5. AE Dunn, D J Dunn, M Lim, C Boyer, N T K Thanh, et al. (2013) Recent developments in the design of nanomaterials for photothermal and magnetic hyperthermia induced controllable drug delivery in Nanoscience 2: 225-254.

6. R Ghosh Chaudhuri, S Paria (2012) Core Shell Nanoparticles: Classes, Properties, Synthesis Mechanisms, Characterization, and Applications Chem Rev 112(4): 2373-2433.

7. A Chichel, J Skowronek, M Kubaszewska, M Kanikowski (2007) Hyperthermia description of a method and a review of clinical applications Rep Pract Oncol Radiother 12(5): 267-275.

8. J E Rosen, L Chan, D B Shieh, F X Gu (2012) Iron oxide nanoparticles for targeted cancer imaging and diagnostics Nanomedicine Nanotechno Biol Med 8(3): 275-290.

9. E Jamal Al Dine, Z Ferjaoui, J Ghanbaja, T Roques Carmes, A Meftah, et al. (2017) Thermo-responsive magnetic Fe304@P(ME02MAXOEGMA100-X) NPs and their applications as drug delivery systems Int J Pharm 532(2): 738-747.

10. J L Vivero Escoto , Y T Huang (2011) Inorganic Organic Hybrid Nanomaterials for Therapeutic and Diagnostic Imaging Applications Int J Mol Sci 12(6): 3888-3927.
11. M Mahmoudi, I Lynch, M R Ejtehadi, M P Monopoli, F B Bombelli, et al. (2011) Protein nanoparticle interactions: opportunities and challenges Chem Rev 111(9): 5610-5637.

12.Z Ferjaoui, R Schneider, A Meftah, E Gaffet, H Alem (2017) Functional responsive superparamagnetic core/shell nanoparticles and their drug release properties RSC Adv 7: 26243-26249.

13. E J Al Dine, S Marchal, R Schneider, B Hamie, J Ghanbaja, et al. (2018) A Facile Approach for Doxorubicine Delivery in Cancer Cells by Responsive and Fluorescent Core Shell Quantum Dots Bioconjug Chem 29(7): 22482256.

14.S Shen, Y Wu, Y Liu, D Wu (2017) High drug-loading nanomedicines: progress, current status, and prospects Int J Nanomedicine 12: 40854109.

15. E J A Dine, Z Ferjaoui, T Roques Carmes, A Schjen, A Meftah, et al. (2017) Nanotechnology 28: 125601.

16.Z Ferjaoui, E Jamal Al Dine, A Kulmukhamedova, L Bezdetnaya, C Soon Chang, et al. (2019) Doxorubicin-Loaded Thermoresponsive Superparamagnetic Nanocarriers for Controlled Drug Delivery and Magnetic Hyperthermia Applications ACS Appl Mater Interfaces 11(34): 30610-30620.

17. I W Mak, N Evaniew, M Ghert (2014) Lost in translation: animal models and clinical trials in cancer treatment Am J Transl Res 6(2): 114-118.

18. M L Shuler, Lab Chip, DOI:10 1039/C8LC90089B.

19. G Kaushik, J Leijten, A Khademhosseini (2017) Concise Review: Organ Engineering: Design, Technology, and Integration STEM CELLS 35(1): 51-60.

20. E W Esch, A Bahinski, D Huh (2015) Organs-on-chips at the frontiers of drug discovery Nat Rev Drug Discov 14(4): 248-260.

21. D E Ingber (2018) Developmentally inspired human 'organs on chips' Development 145(16) dev156125.

22. B Zhang, M Radisic (2017) Organ on a chip device advance to market Lab Chip 17(14): 2395-2420.

23. S N Bhatia, D E Ingber (2014) Microfluidic organs on chips Nat Biotechnol 32(8): 760-772.

24. T Pasman, D Grijpma, D Stamatialis , A Poot (2018) Flat and microstructured polymeric membranes in organs on chips $\mathrm{J}$ R Soc Interface 15(144): 20180351

25. X Yang, K Li, X Zhang, C Liu, B Guo, et al. (2018) Nanofiber membrane supported lung on a chip microdevice for anti-cancer drug testing Lab Chip 18(3) 486-495.

26. V M Gaspar, E C Costa, J A Queiroz, C Pichon, F Sousa et al. (2015) Folate targeted multifunctional amino acid chitosan nanoparticles for improved cancer therapy Pharm Res 32(2): 562-577.

27. J Barthes, N E Vrana, H Ozçelik, R Gahoual, Y N François, et al. (2015) Priming cells for their final destination: microenvironment controlled cell culture by a modular ECM mimicking feeder film Biomater Sci 3(9): 1302-1311.

28. V H Nguyen, B J Lee (2017) Protein corona: a new approach for nanomedicine design Int J Nanomedicine 12: 3137-3151.

29. (2018) Wyss Institut.

30. D Huh, B D Matthews, A Mammoto, M Montoya Zavala, H Y Hsin, et al. (2010) Reconstituting organ-level lung functions on a chip Science 328(5986):1662-1668. 
ISSN: 2574-1241

DOI: 10.26717/BJSTR.2019.23.003841

Halima Alem. Biomed J Sci \& Tech Res

(c) (P) This work is licensed under Creative

Submission Link: https://biomedres.us/submit-manuscript.php

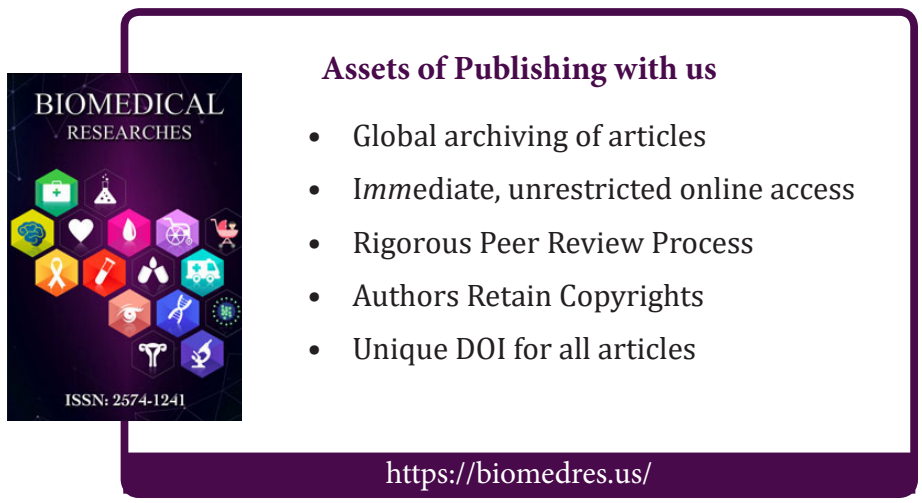

